\title{
A New $C$-Eigenvalue Localisation Set for Piezoelectric-Type Tensors
}

\author{
Liang Xiong and Jianzhou Liu* \\ School of Mathematics and Computational Science, Xiangtan University, Xiangtan \\ 411105, Hunan, P.R. China.
}

Received 6 January 2019; Accepted (in revised version) 4 June 2019.

\begin{abstract}
A new inclusion set for localisation of the $C$-eigenvalues of piezoelectric tensors is established. Numerical experiments show that it is better or comparable to the methods known in literature.
\end{abstract}

AMS subject classifications: 15A18, 15A69, 15A21

Key words: $C$-eigenvalue, $C$-eigenvector, piezoelectric tensor, $C$-eigenvalue localisation theorem.

\section{Introduction}

Third order tensors play an important role in physics and engineering, including nonlinear optics $[10,12]$, properties of crystals $[6,11,19,20,22,26]$ and liquid crystals $[5,9,24]$. In particular, piezoelectric tensors find wide applications in converse piezoelectric and piezoelectric effects [4]. Chen et al. [4] specify the piezoelectric-type tensors as follows.

Definition 1.1 (cf. Chen et al. [4]). A third order $n$-dimensional tensor $\mathscr{A}=\left(a_{i j k}\right) \in \mathbb{R}^{n \times n \times n}$ is called the piezoelectric-type tensor if the last two indices of $\mathscr{A}$ are symmetric - i.e. if $a_{i j k}=a_{i k j}$ for all $j, k \in[n]$, where $[n]:=\{1,2, \ldots, n\}$.

Qi [21] and Lim [18] introduced the notion of eigenvalues for higher order tensors. It is worth noting that the eigenvalues of the third order symmetric traceless-tensors are widely used in the theory of liquid crystals [5,9,24]. Following these ideas, Chen et al. [4] defined $C$-eigenvalues and $C$-eigenvectors for piezoelectric-type tensors, which turn out to be useful in the study of piezoelectric and converse piezoelectric effects in solid crystals.

Definition 1.2 (cf. Chen et al. [4]). Let $\mathscr{A}=\left(a_{i j k}\right) \in \mathbb{R}^{n \times n \times n}$ be a third-order $n$-dimensional tensor. A number $\lambda \in \mathbb{R}$ is called the $C$-eigenvalue of $\mathscr{A}$ if there are $x, y \in \mathbb{R}^{n}$ such that

$$
\mathscr{A} y y=\lambda x, \quad x \mathscr{A} y=\lambda y, \quad x^{\top} x=1, \quad y^{\top} y=1,
$$

*Corresponding author. Email addresses: liujz@xtu.edu.cn (J. Liu) 
where

$$
(\mathscr{A} y y)_{i}=\sum_{k, j \in[n]} c_{i k j} y_{k} y_{j}, \quad(x \mathscr{A} y)_{i}=\sum_{k, j \in[n]} c_{k j i} x_{k} y_{j}
$$

The vectors $x$ and $y$ are referred to as associated left and right $C$-eigenvectors, respectively.

By $\sigma(\mathscr{A})$ we denote the $C$-spectrum of the piezoelectric-type tensor $\mathscr{A}$-i.e. the set of all $C$-eigenvalues of the piezoelectric-type tensor $\mathscr{A}$. The $C$-spectral radius of $\mathscr{A}$ is defined by

$$
\rho(\mathscr{A}):=\max \{|\lambda|: \lambda \in \sigma(\mathscr{A})\} .
$$

For a piezoelectric tensor $\mathscr{A}$, Chen et al. [4] proved the existence of $C$-eigenvalues associated with left and right $C$-eigenvectors. They also showed that the largest $C$-eigenvalue of the piezoelectric tensor represents the highest piezoelectric coupling constant and it can be determined as

$$
\lambda^{*}=\max \left\{x \mathscr{A} y y: x^{\top} x=1, y^{\top} y=1\right\}
$$

where

$$
x \mathscr{A} y y:=\sum_{i, k, j \in[n]} c_{i j k} x_{i} y_{j} y_{k} .
$$

However, the practical calculation of $\lambda^{*}$ is a challenging problem because of the uncertainty with the $C$-eigenvectors $x$ and $y$ in actual operations. On the other hand, we can capture all eigenvalues of a high order tensor by the eigenvalue localisation. In particular, for real symmetric tensors, Qi [21] considers an eigenvalue localisation set, which is an extension of the Geršgorin matrix eigenvalue inclusion theorem for matrices [23]. For general tensors, Li et al. [16] proposed Brauer-type eigenvalue inclusion sets. Later on, various eigenvalue localisation sets and their applications have been studied in Refs. $[1,2,8,13,14,17,25,27]$.

Recently, C. Li and Y. Li [15] introduced two intervals to estimate all $C$-eigenvalues of a piezoelectric-type tensor.

Theorem 1.1 (cf. C. Li \& Y. Li [15]). If $\lambda$ is a C-eigenvalue of the piezoelectric-type tensor $\mathscr{C}=\left(c_{i j k}\right) \in \mathbb{R}^{n \times n \times n}$, then

$$
\lambda \in[-\rho, \rho],
$$

where

$$
\begin{aligned}
& \rho=\max _{i, j \in[n]}\left\{R_{i}^{(1)}(\mathscr{C}) R_{j}(\mathscr{C})\right\}^{1 / 2}, \\
& R_{i}^{(1)}(\mathscr{C})=\sum_{l, k \in[n]}\left|c_{i l k}\right|, R_{j}(\mathscr{C})=\sum_{l, k \in[n]}\left|c_{l k j}\right|, \quad[n]=\{1,2, \ldots, n\} .
\end{aligned}
$$

Theorem 1.2 (cf. C. Li \& Y. Li [15]). If $\lambda$ is a C-eigenvalue of the piezoelectric-type tensor $\mathscr{C}=\left(c_{i j k}\right) \in \mathbb{R}^{n \times n \times n}$ and $S$ is a subset of $[n]$, then

$$
\lambda \in\left[-\rho_{s}, \rho_{s}\right],
$$


where

$$
\begin{aligned}
& \rho_{S}=\max _{i, j \in[n]} \frac{1}{2}\left\{R_{j}^{\Delta_{S}}(\mathscr{C})+\left(R_{j}^{\Delta_{S}}(\mathscr{C})\right)^{2}+4 R_{i}^{(1)}(\mathscr{C})\left(R_{j}^{\bar{\Delta}_{S}}(\mathscr{C})\right)^{1 / 2}\right\}, \\
& \Delta_{S}=\{(i, j): i \in S \text { or } j \in S\}, \quad \bar{\Delta}_{S}=\{(i, j): i \notin S \text { and } j \notin S\},
\end{aligned}
$$

and

$$
R_{j}^{\Delta_{S}}(\mathscr{C})=\sum_{l, k \in \Delta_{S}}\left|c_{l k j}\right|, R_{j}^{\bar{\Delta}_{S}}(\mathscr{C})=\sum_{l, k \in \bar{\Delta}_{S}}\left|c_{l k j}\right|
$$

Moreover,

$$
\lambda \in\left[-\rho_{\min }, \rho_{\min }\right],
$$

where $\rho_{\min }=\min _{S \subseteq[n]} \rho_{S}$.

Theorem 1.3 (cf. C. Li \& Y. Li [15]). If $\lambda$ is a C-eigenvalue of the piezoelectric-type tensor $\mathscr{C}=\left(c_{i j k}\right) \in \mathbb{R}^{n \times n \times n}$, then

$$
\lambda \in\left[-\rho_{\min }, \rho_{\min }\right] \subseteq[-\rho, \rho],
$$

where $\rho$ and $\rho_{\min }$ are defined in Theorems 1.1 and 1.2, respectively.

On the other hand, Che et al. [3] proposed another localisation set for $C$-eigenvalues.

Theorem 1.4 (cf. Che et al. [3]). Let $\mathscr{C}=\left(c_{i j k}\right) \in \mathbb{R}^{n \times n \times n}$ be a piezoelectric-type tensor. Then

$$
\sigma(\mathscr{C}) \subseteq \Gamma(\mathscr{C})=\bigcup_{j \in[n]} \Gamma_{j}(\mathscr{C}),
$$

where $\Gamma_{j}(\mathscr{C})=\left\{z \in \mathbb{C} ;|z| \leq R_{j}(\mathscr{C})\right\}$ and $R_{j}(\mathscr{C})=\sum_{l, k \in[n]}\left|c_{l k j}\right|$.

Theorem 1.5 (cf. Che et al. [3]). If $\mathscr{C}=\left(c_{i j k}\right) \in \mathbb{R}^{n \times n \times n}$ is a piezoelectric-type tensor, then

$$
\sigma(\mathscr{C}) \subseteq \mathscr{L}(\mathscr{C})=\bigcup_{j \in[n]}\left(\bigcap_{k \in[n], k \neq j} \mathscr{L}_{j, k}(\mathscr{C})\right),
$$

where

$$
\begin{aligned}
& \quad \mathscr{L}_{j, k}(\mathscr{C})=\left\{z \in \mathbb{C}:\left(|z|-R_{j}(\mathscr{C})+R_{j}^{k}(\mathscr{C})\right)|z| \leq R_{j}^{k}(\mathscr{C}) R_{k}(\mathscr{C})\right\} \\
& \text { and } R_{j}^{k}(\mathscr{C})=\sum_{l \in[n]}\left|c_{l k j}\right| .
\end{aligned}
$$

Theorem 1.6 (cf. Che et al. [3]). Let $\mathscr{C}=\left(c_{i j k}\right) \in \mathbb{R}^{n \times n \times n}$ be a piezoelectric-type tensor. Then

$$
\sigma(\mathscr{C}) \subseteq \mathscr{M}(\mathscr{C})=\bigcup_{i, k \in[n], k \neq i}\left(\mathscr{M}_{i, k}(\mathscr{C}) \bigcup \mathscr{H}_{i, k}(\mathscr{C})\right)
$$

where

$$
\mathscr{M}_{i, k}(\mathscr{C})=\left\{z \in \mathbb{C}:\left(|z|-\left(R_{i}(\mathscr{C})-R_{i}^{k}(\mathscr{C})\right)\right)\left(|z|-R_{k}^{k}(\mathscr{C})\right) \leq R_{i}^{k}(\mathscr{C})\left(R_{k}(\mathscr{C})-R_{k}^{k}(\mathscr{C})\right)\right\}
$$

and

$$
\mathscr{H}_{i, k}(\mathscr{C})=\left\{z \in \mathbb{C}:|z|-\left(R_{i}(\mathscr{C})-R_{i}^{k}(\mathscr{C})\right) \leq 0,|z|-R_{k}^{k}(\mathscr{C}) \leq 0\right\}
$$


Comparing the sets above, one can show that $\mathscr{L}(\mathscr{C}) \subseteq \Gamma(\mathscr{C})$ and $\mathscr{M}(\mathscr{C}) \subseteq \Gamma(\mathscr{C})$, i.e. the sets $\mathscr{L}(\mathscr{C})$ and $\mathscr{M}(\mathscr{C})$ are more tight than $\Gamma(\mathscr{C})$.

In this work, we present a new $C$-eigenvalue inclusion set, which is more accurate than the set $\Gamma(\mathscr{C})$ in Theorem 1.4. Moreover, numerical examples show that in some cases, it is superior to all the sets $\Gamma(\mathscr{C}), \mathscr{M}(\mathscr{C})$ and $\mathscr{L}(\mathscr{C})$.

\section{New $C$-Eigenvalue Localisation Sets}

In this section, we propose a new localisation set for $C$-eigenvalues and establish the relationship between this set and the set $\Gamma(\mathscr{C})$ from Theorem 1.4.

Theorem 2.1. If $\mathscr{C}=\left(c_{i j k}\right) \in \mathbb{R}^{n \times n \times n}$ is a piezoelectric-type tensor, then

$$
\sigma(\mathscr{C}) \subseteq \Upsilon(\mathscr{C})=\bigcup_{i, k \in[n], k \neq i}\left(\hat{\Upsilon}_{i, k}(\mathscr{C}) \bigcup \tilde{\Upsilon}_{i, k}(\mathscr{C})\right)
$$

where

$$
\hat{\Upsilon}_{i, k}(\mathscr{C})=\left\{z \in \mathbb{R}:|z|-R_{i}(\mathscr{C})+R_{i}^{k}(\mathscr{C}) \leq 0,|z|-R_{k}(\mathscr{C})+R_{k}^{i}(\mathscr{C}) \leq 0\right\}
$$

and

$$
\tilde{\Upsilon}_{i, k}(\mathscr{C})=\left\{z \in \mathbb{R}:\left[|z|-R_{i}(\mathscr{C})+R_{i}^{k}(\mathscr{C})\right]\left[|z|-R_{k}(\mathscr{C})+R_{k}^{i}(\mathscr{C})\right] \leq R_{i}^{k}(\mathscr{C}) R_{k}^{i}(\mathscr{C})\right\} .
$$

Proof. Consider a $C$-eigenvalue $\lambda$ of $\mathscr{C}$ with corresponding left $C$-eigenvector $x=$ $\left(x_{1}, x_{2}, \ldots, x_{n}\right)^{\top}$ and right $C$-eigenvector $y=\left(y_{1}, y_{2}, \ldots, y_{n}\right)^{\top}$, so that

$$
\mathscr{C} y y=\lambda x, \quad x \mathscr{C} y=\lambda y, \quad x^{\top} x=1, \quad y^{\top} y=1 .
$$

The assumption

$$
\left|y_{p}\right| \geq\left|y_{q}\right| \geq \max _{i \in N, i \neq p, q}\left|y_{i}\right|
$$

yields $0<\left|y_{p}\right| \leq 1$. If follows from (2.1) that

$$
\lambda y_{p}=\sum_{l, k \in[n]} c_{l k p} x_{l} y_{k}=\sum_{\substack{l, k \in[n], k \neq q}} c_{l k p} x_{l} y_{k}+\sum_{l \in[n]} c_{l q p} x_{l} y_{q} .
$$

Since $0 \leq\left|x_{i}\right| \leq 1$ for any $i \in[n]$, we obtain

$$
|\lambda| \leq \sum_{\substack{l, k \in[n], k \neq q}}\left|c_{l k p}\right| \frac{\left|y_{k}\right|}{\left|y_{p}\right|}+\sum_{l \in[n]}\left|c_{l q p}\right| \frac{\left|y_{q}\right|}{\left|y_{p}\right|} \leq \sum_{\substack{l, k \in[n], k \neq q}}\left|c_{l k p}\right|+\sum_{l \in[n]}\left|c_{l q p}\right| \frac{\left|y_{q}\right|}{\left|y_{p}\right|} .
$$

Writing the last inequality as

$$
|\lambda|-\sum_{\substack{l, k \in[n], k \neq q}}\left|c_{l k p}\right| \leq \sum_{l \in[n]}\left|c_{l q p}\right| \frac{\left|y_{q}\right|}{\left|y_{p}\right|}
$$

we obtain the following estimates: 
1. If $\left|y_{q}\right|=0$, then $|\lambda|-\sum_{\substack{l, k \in[n], k \neq q}}\left|c_{l k p}\right| \leq 0$.

2. If $|\lambda|-R_{q}(\mathscr{C})+R_{q}^{p}(\mathscr{C}) \geq 0$, then $\lambda \in \tilde{\Upsilon}_{p, q}(\mathscr{C})$.

3. If $|\lambda|-R_{q}(\mathscr{C})+R_{q}^{p}(\mathscr{C}) \leq 0$, then $\lambda \in \hat{\Upsilon}_{p, q}(\mathscr{C})$.

On the other hand, if $\left|y_{q}\right|>0$, then

$$
\lambda y_{q}=\sum_{l, k \in[n]} c_{l k q} x_{l} y_{k}=\sum_{\substack{l, k \in[n], k \neq p}} c_{l k q} x_{l} y_{k}+\sum_{l \in[n]} c_{l p q} x_{l} y_{p},
$$

and

$$
|\lambda| \leq \sum_{\substack{l, k \in[n], k \neq p}}\left|c_{l k q}\right| \frac{\left|y_{k}\right|}{\left|y_{q}\right|}+\sum_{l \in[n]}\left|c_{l p q}\right| \frac{\left|y_{p}\right|}{\left|y_{q}\right|} \leq \sum_{\substack{l, k \in[n], k \neq p}}\left|c_{l k q}\right|+\sum_{l \in[n]}\left|c_{l p q}\right| \frac{\left|y_{p}\right|}{\left|y_{q}\right|} .
$$

Writing it in the form

$$
|\lambda|-\sum_{\substack{l, k \in[n], k \neq p}}\left|c_{l k q}\right| \leq \sum_{l \in[n]}\left|c_{l p q}\right| \frac{\left|y_{p}\right|}{\left|y_{q}\right|}
$$

we note that if $|\lambda|-\sum_{\substack{l, k \in[n], k \neq q}}\left|c_{l k p}\right| \leq 0$ or $|\lambda|-R_{q}(\mathscr{C})+R_{q}^{p}(\mathscr{C}) \leq 0$, then multiplying (2.2) and (2.3), we arrive at the inequality

$$
\left(|\lambda|-\sum_{\substack{l, k \in[n], k \neq q}}\left|c_{l k p}\right|\right)\left(|\lambda|-\sum_{\substack{l, k \in[n], k \neq p}}\left|c_{l k q}\right|\right) \leq \sum_{l \in[n]}\left|c_{l q p}\right| \sum_{l \in[n]}\left|c_{l p q}\right| .
$$

It can be written as

$$
\left[|\lambda|-R_{p}(\mathscr{C})+R_{p}^{q}(\mathscr{C})\right]\left[|\lambda|-R_{q}(\mathscr{C})+R_{q}^{p}(\mathscr{C})\right] \leq R_{p}^{q}(\mathscr{C}) R_{q}^{p}(\mathscr{C}),
$$

so that $\lambda \in \tilde{\Upsilon}_{p, q}(\mathscr{C}) \subseteq \Upsilon(\mathscr{C})$. If

$$
|\lambda|-\sum_{\substack{l, k \in[n], k \neq q}}\left|c_{l k p}\right| \leq 0 \quad \text { and } \quad|\lambda|-R_{q}(\mathscr{C})+R_{q}^{p}(\mathscr{C}) \leq 0,
$$

then $\lambda \in \hat{\Upsilon}_{p, q}(\mathscr{C}) \subseteq \Upsilon(\mathscr{C})$, as required.

Remark 2.1. For a real tensor $\mathscr{C}=\left(c_{i j k}\right) \in \mathbb{R}^{n \times n \times n}, n \geq 2$, the set $\Gamma(\mathscr{C})$ consists of $n$ sets $\Gamma_{j}(\mathscr{C})$, the set $\mathscr{L}(\mathscr{C})$ of $n(n-1)$ sets $\mathscr{L}_{j, k}(\mathscr{C})$, the set $\mathscr{M}(\mathscr{C})$ of $n(n-1)$ sets $\mathscr{M}_{i, k}(\mathscr{C})$ and $n(n-1)$ sets $\mathscr{H}_{i, k}(\mathscr{C})$, and the set $\Upsilon(\mathscr{C})$ of $n(n-1)$ sets $\hat{\Upsilon}_{i, k}(\mathscr{C})$ and $n(n-1)$ sets $\tilde{\Upsilon}_{i, k}(\mathscr{C})$. It is worth noting that for large $n$, the set $\Upsilon(\mathscr{C})$ locates all eigenvalues of $\mathscr{C}$ more accurately than $\Gamma(\mathscr{C})$, but $\Gamma(\mathscr{C})$ can be determined with less computational effort. 
Comparing the sets $\Gamma(\mathscr{C})$ and $\Upsilon(\mathscr{C})$, we obtain the following result.

Theorem 2.2. If $\mathscr{C}=\left(c_{i j k}\right) \in \mathbb{R}^{n \times n \times n}$ is a piezoelectric-type tensor, then

$$
\sigma(\mathscr{C}) \subseteq \Upsilon(\mathscr{C}) \subseteq \Gamma(\mathscr{C})
$$

Proof. For any $z \in \Upsilon(\mathscr{C})$, there exist $i, k \in[n], i \neq k$ such that $z \in \hat{\Upsilon}_{i, k}(\mathscr{C})$ or $z \in \tilde{\Upsilon}_{i, k}(\mathscr{C})$. Next, we consider two cases.

Case I. If $z \in \hat{\Upsilon}_{i, k}(\mathscr{C})$, i.e. if

$$
|z|-R_{i}(\mathscr{C})+R_{i}^{k}(\mathscr{C}) \leq 0 \quad \text { and } \quad|z|-R_{k}(\mathscr{C})+R_{k}^{i}(\mathscr{C}) \leq 0,
$$

then

$$
|z| \leq R_{i}(\mathscr{C}) \text { and }|z| \leq R_{k}(\mathscr{C})
$$

so that $z \in \Gamma(\mathscr{C})$.

Case II. If $z \in \tilde{\Upsilon}_{i, k}(\mathscr{C})$, then

$$
\left[|z|-R_{i}(\mathscr{C})+R_{i}^{k}(\mathscr{C})\right]\left[|z|-R_{k}(\mathscr{C})+R_{k}^{i}(\mathscr{C})\right] \leq R_{i}^{k}(\mathscr{C}) R_{k}^{i}(\mathscr{C}) .
$$

Assuming first that $R_{i}^{k}(\mathscr{C}) R_{k}^{i}(\mathscr{C})=0$, we obtain

$$
|z|-R_{i}(\mathscr{C})+R_{i}^{k}(\mathscr{C}) \leq 0 \quad \text { or } \quad|z|-R_{k}(\mathscr{C})+R_{k}^{i}(\mathscr{C}) \leq 0 .
$$

It follows that

$$
|z| \leq R_{i}(\mathscr{C}) \text { or }|z| \leq R_{k}(\mathscr{C})
$$

Hence, $z \in \Gamma(\mathscr{C})$.

If we now assume that $R_{i}^{k}(\mathscr{C}) R_{k}^{i}(\mathscr{C})>0$, then (2.4) yields

$$
\frac{|z|-R_{i}(\mathscr{C})+R_{i}^{k}(\mathscr{C})}{R_{i}^{k}(\mathscr{C})} \cdot \frac{|z|-R_{k}(\mathscr{C})+R_{k}^{i}(\mathscr{C})}{R_{k}^{i}(\mathscr{C})} \leq 1,
$$

and at least one of the inequalities

$$
\frac{|z|-R_{i}(\mathscr{C})+R_{i}^{k}(\mathscr{C})}{R_{i}^{k}(\mathscr{C})} \leq 1, \quad \frac{|z|-R_{k}(\mathscr{C})+R_{k}^{i}(\mathscr{C})}{R_{k}^{i}(\mathscr{C})} \leq 1
$$

holds. It follows that $z \in \Gamma_{i}(\mathscr{C}) \bigcup \Gamma_{k}(\mathscr{C})$ and combining both cases, we finish the proof.

\section{Numerical Examples}

In this section, we provide the results of numerical experiments to show that our approach locates $C$-eigenvalues much better than other methods. The piezoelectric tensors used in the examples, arise in piezoelectric materials with symmetries and have been previously studied in Refs. [3, 4, 7, 22]. 
Example 3.1. Consider the piezoelectric tensor $\mathscr{A}_{V F e S b}$ [7] with the entries

$$
a_{i j k}=\left\{\begin{array}{l}
a_{123}=a_{213}=a_{312}=-3.68180667, \\
a_{i j k}, \quad \text { otherwise. }
\end{array}\right.
$$

According to [4], the largest $C$-eigenvalue of $\mathscr{A}_{V F e S b}$ is about 4.25138 and Theorems 1.4-1.6 show that

$$
\begin{aligned}
\Gamma(\mathscr{C}) & =\bigcup_{j \in[n]} \Gamma_{j}(\mathscr{C})=\{z \in \mathbb{C}:|z| \leq 7.3636\}, \\
\mathscr{L}(\mathscr{C}) & =\bigcup_{j \in[n]}\left(\bigcap_{k \in[n], k \neq j} \mathscr{L}_{j, k}(\mathscr{C})\right)=\{z \in \mathbb{C}:|z| \leq 7.3636\}, \\
\mathscr{M}(\mathscr{C}) & =\bigcup_{i, k \in[n], k \neq i}\left(\mathscr{M}_{i, k}(\mathscr{C}) \bigcup \mathscr{H}_{i, k}(\mathscr{C})\right)=\{z \in \mathbb{C}:|z| \leq 7.3636\} .
\end{aligned}
$$

From Theorem 2.1, we get

$$
\Upsilon(\mathscr{C})=\bigcup_{i, k \in[n], k \neq i}\left(\hat{\Upsilon}_{i, k}(\mathscr{C}) \bigcup \tilde{\Upsilon}_{i, k}(\mathscr{C})\right)=\{z \in \mathbb{C}:|z| \leq 7.3636\}
$$

Example 3.2. Consider the piezoelectric tensor $\mathscr{A}_{\mathrm{SiO} 2}[6,22]$ with the entries

$$
a_{i j k}=\left\{\begin{array}{l}
a_{111}=-a_{122}=a_{212}=-0.13685, \\
a_{123}=-a_{213}=-0.009715, \\
a_{i j k}, \quad \text { otherwise. }
\end{array}\right.
$$

According to [4], the largest $C$-eigenvalue of $\mathscr{A}_{\mathrm{SiO} 2}$ is about 0.1375 and Theorems 1.41.6 show that

$$
\begin{aligned}
\Gamma(\mathscr{C}) & =\bigcup_{j \in[n]} \Gamma_{j}(\mathscr{C})=\{z \in \mathbb{C}:|z| \leq 0.2834\} \\
\mathscr{L}(\mathscr{C}) & =\bigcup_{j \in[n]}\left(\bigcap_{k \in[n], k \neq j} \mathscr{L}_{j, k}(\mathscr{C})\right)=\{z \in \mathbb{C}:|z| \leq 0.2744\}, \\
\mathscr{M}(\mathscr{C}) & =\bigcup_{i, k \in[n], k \neq i}\left(\mathscr{M}_{i, k}(\mathscr{C}) \bigcup \mathscr{H}_{i, k}(\mathscr{C})\right)=\{z \in \mathbb{C}:|z| \leq 0.2834\} .
\end{aligned}
$$

From Theorem 2.1, we have

$$
\Upsilon(\mathscr{C})=\bigcup_{i, k \in[n], k \neq i}\left(\hat{\Upsilon}_{i, k}(\mathscr{C}) \bigcup \tilde{\Upsilon}_{i, k}(\mathscr{C})\right)=\{z \in \mathbb{C}:|z| \leq 0.2834\}
$$

Example 3.3. Consider the piezoelectric tensor $\mathscr{A}_{C r 2 \mathrm{AgBiO8}}$ [7] with the entries

$$
a_{i j k}=\left\{\begin{array}{l}
a_{123}=a_{213}=-0.22163, \\
a_{113}=-a_{223}=2.608665, \\
a_{311}=-a_{322}=0.152485 \\
a_{312}=-0.37153 \\
a_{i j k}, \quad \text { otherwise }
\end{array}\right.
$$


According to [4], the largest $C$-eigenvalue of $\mathscr{A}_{C r 2 A g B i O 8}$ is about 2.6258 and Theorems 1.4-1.6 show that

$$
\begin{aligned}
\Gamma(\mathscr{C}) & =\bigcup_{j \in[n]} \Gamma_{j}(\mathscr{C})=\{z \in \mathbb{C}:|z| \leq 5.6606\}, \\
\mathscr{L}(\mathscr{C}) & =\bigcup_{j \in[n]}\left(\bigcap_{k \in[n], k \neq j} \mathscr{L}_{j, k}(\mathscr{C})\right)=\{z \in \mathbb{C}:|z| \leq 4.8058\}, \\
\mathscr{M}(\mathscr{C}) & =\bigcup_{i, k \in[n], k \neq i}\left(\mathscr{M}_{i, k}(\mathscr{C}) \bigcup \mathscr{H}_{i, k}(\mathscr{C})\right)=\{z \in \mathbb{C}:|z| \leq 4.7861\} .
\end{aligned}
$$

From Theorem 2.1, we have

$$
\Upsilon(\mathscr{C})=\bigcup_{i, k \in[n], k \neq i}\left(\hat{\Upsilon}_{i, k}(\mathscr{C}) \bigcup \tilde{\Upsilon}_{i, k}(\mathscr{C})\right)=\{z \in \mathbb{C}:|z| \leq 4.7335\}
$$

Example 3.4. Consider the piezoelectric tensor $\mathscr{A}_{\mathrm{RbTaO3}}$ [7] with the entries

$$
a_{i j k}=\left\{\begin{array}{l}
a_{113}=a_{223}=-8.40955 \\
a_{222}=-a_{212}=-a_{211}=-5.412525 \\
a_{311}=-a_{322}=-4.3031 \\
a_{333}=-5.14766 \\
a_{i j k}, \quad \text { otherwise. }
\end{array}\right.
$$

According to [4], the largest $C$-eigenvalue of $\mathscr{A}_{\mathrm{RbTaO}}$ is about 12.4234 and Theorems 1.4-1.6 show that

$$
\begin{aligned}
\Gamma(\mathscr{C}) & =\bigcup_{j \in[n]} \Gamma_{j}(\mathscr{C})=\{z \in \mathbb{C}:|z| \leq 23.5377\}, \\
\mathscr{L}(\mathscr{C}) & =\bigcup_{j \in[n]}\left(\bigcap_{k \in[n], k \neq j} \mathscr{L}_{j, k}(\mathscr{C})\right)=\{z \in \mathbb{C}:|z| \leq 23.5377\}, \\
\mathscr{M}(\mathscr{C}) & =\bigcup_{i, k \in[n], k \neq i}\left(\mathscr{M}_{i, k}(\mathscr{C}) \bigcup \mathscr{H}_{i, k}(\mathscr{C})\right)=\{z \in \mathbb{C}:|z| \leq 23.5377\} .
\end{aligned}
$$

From Theorem 2.1, we have

$$
\Upsilon(\mathscr{C})=\bigcup_{i, k \in[n], k \neq i}\left(\hat{\Upsilon}_{i, k}(\mathscr{C}) \bigcup \tilde{\Upsilon}_{i, k}(\mathscr{C})\right)=\{z \in \mathbb{C}:|z| \leq 23.5377\} .
$$

Example 3.5. Consider the piezoelectric tensor $\mathscr{A}_{\text {NaBiS2 }}$ [7] with the entries

$$
a_{i j k}=\left\{\begin{array}{l}
a_{113}=-8.90808, a_{223}=-0.00842, a_{311}=-7.11526 \\
a_{322}=-0.6222, a_{333}=-7.93831 \\
a_{i j k}, \quad \text { otherwise }
\end{array}\right.
$$


According to [4], the largest $C$-eigenvalue of $\mathscr{A}_{\mathrm{NaBiS2}}$ is about 11.6674 and Theorems 1.4-1.6 show that

$$
\begin{aligned}
\Gamma(\mathscr{C}) & =\bigcup_{j \in[n]} \Gamma_{j}(\mathscr{C})=\{z \in \mathbb{C}:|z| \leq 16.8548\}, \\
\mathscr{L}(\mathscr{C}) & =\bigcup_{j \in[n]}\left(\bigcap_{k \in[n], k \neq j} \mathscr{L}_{j, k}(\mathscr{C})\right)=\{z \in \mathbb{C}:|z| \leq 16.5640\}, \\
\mathscr{M}(\mathscr{C}) & =\bigcup_{i, k \in[n], k \neq i}\left(\mathscr{M}_{i, k}(\mathscr{C}) \bigcup \mathscr{H}_{i, k}(\mathscr{C})\right)=\{z \in \mathbb{C}:|z| \leq 16.8464\} .
\end{aligned}
$$

From Theorem 2.1, we have

$$
\Upsilon(\mathscr{C})=\bigcup_{i, k \in[n], k \neq i}\left(\hat{\Upsilon}_{i, k}(\mathscr{C}) \bigcup \tilde{\Upsilon}_{i, k}(\mathscr{C})\right)=\{z \in \mathbb{C}:|z| \leq 16.8464\}
$$

Example 3.6. Consider the piezoelectric tensor $\mathscr{A}_{\text {LiBiB2O5 }}$ [7] with the entries

$$
a_{i j k}=\left\{\begin{array}{l}
a_{123}=2.35682, a_{112}=0.34929, a_{211}=0.16101, a_{222}=0.12562 \\
a_{233}=0.1361, a_{213}=-0.05587, a_{323}=6.91074, a_{312}=2.57812 \\
a_{i j k}, \quad \text { otherwise }
\end{array}\right.
$$

According to [4], the largest $C$-eigenvalue of $\mathscr{A}_{\text {LiBiB2O5 }}$ is about 7.7376 and Theorems 1.4-1.6 show that

$$
\begin{aligned}
\Gamma(\mathscr{C}) & =\bigcup_{j \in[n]} \Gamma_{j}(\mathscr{C})=\{z \in \mathbb{C}:|z| \leq 12.3206\}, \\
\mathscr{L}(\mathscr{C}) & =\bigcup_{j \in[n]}\left(\bigcap_{k \in[n], k \neq j} \mathscr{L}_{j, k}(\mathscr{C})\right)=\{z \in \mathbb{C}:|z| \leq 11.0127\}, \\
\mathscr{M}(\mathscr{C}) & =\bigcup_{i, k \in[n], k \neq i}\left(\mathscr{M}_{i, k}(\mathscr{C}) \bigcup \mathscr{H}_{i, k}(\mathscr{C})\right)=\{z \in \mathbb{C}:|z| \leq 11.0038\} .
\end{aligned}
$$

From Theorem 2.1, we have

$$
\Upsilon(\mathscr{C})=\bigcup_{i, k \in[n], k \neq i}\left(\hat{\Upsilon}_{i, k}(\mathscr{C}) \bigcup \tilde{\Upsilon}_{i, k}(\mathscr{C})\right)=\{z \in \mathbb{C}:|z| \leq 10.9998\} .
$$

Example 3.7. Consider the piezoelectric tensor $\mathscr{A}_{K B i 2 F 7}[7]$ with the entries

$$
a_{i j k}=\left\{\begin{array}{l}
a_{111}=12.64393, a_{122}=1.08802, a_{133}=4.14350, a_{123}=1.59052, \\
a_{113}=1.96801, a_{112}=0.22465, a_{211}=2.59187, a_{222}=0.08263, \\
a_{233}=0.81041, a_{223}=0.51165, a_{213}=0.71432, a_{212}=0.10570, \\
a_{311}=1.51254, a_{322}=0.68235, a_{333}=-0.23019, a_{323}=0.19013, \\
a_{313}=0.39030, a_{312}=0.08381 .
\end{array}\right.
$$


According to [4], the largest $C$-eigenvalue of $\mathscr{A}_{K B i 2 F 7}$ is about 20.2351 and Theorems 1.4-1.6 show that

$$
\begin{aligned}
\Gamma(\mathscr{C}) & =\bigcup_{j \in[n]} \Gamma_{j}(\mathscr{C})=\{z \in \mathbb{C}:|z| \leq 20.2351\} \\
\mathscr{L}(\mathscr{C}) & =\bigcup_{j \in[n]}\left(\bigcap_{k \in[n], k \neq j} \mathscr{L}_{j, k}(\mathscr{C})\right)=\{z \in \mathbb{C}:|z| \leq 18.8793\} \\
\mathscr{M}(\mathscr{C}) & =\bigcup_{i, k \in[n], k \neq i}\left(\mathscr{M}_{i, k}(\mathscr{C}) \bigcup \mathscr{H}_{i, k}(\mathscr{C})\right)=\{z \in \mathbb{C}:|z| \leq 19.8830\}
\end{aligned}
$$

From Theorem 2.1, we have

$$
\Upsilon(\mathscr{C})=\bigcup_{i, k \in[n], k \neq i}\left(\hat{\Upsilon}_{i, k}(\mathscr{C}) \bigcup \tilde{\Upsilon}_{i, k}(\mathscr{C})\right)=\{z \in \mathbb{C}:|z| \leq 19.8319\} .
$$

Example 3.8. Consider the piezoelectric tensor $\mathscr{A}_{\mathrm{BaNiO3}}$ [7] with the entries

$$
a_{i j k}=\left\{\begin{array}{l}
a_{113}=a_{223}=0.038385, a_{311}=a_{322}=6.89822, a_{333}=27.4628, \\
a_{i j k}, \quad \text { otherwise. }
\end{array}\right.
$$

According to [4], the largest $C$-eigenvalue of $\mathscr{A}_{\mathrm{BaNiO3}}$ is about 27.4628 and Theorems 1.4-1.6 show that

$$
\begin{aligned}
\Gamma(\mathscr{C}) & =\bigcup_{j \in[n]} \Gamma_{j}(\mathscr{C})=\{z \in \mathbb{C}:|z| \leq 27.5396\}, \\
\mathscr{L}(\mathscr{C}) & =\bigcup_{j \in[n]}\left(\bigcap_{k \in[n], k \neq j} \mathscr{L}_{j, k}(\mathscr{C})\right)=\{z \in \mathbb{C}:|z| \leq 27.5109\}, \\
\mathscr{M}(\mathscr{C}) & =\bigcup_{i, k \in[n], k \neq i}\left(\mathscr{M}_{i, k}(\mathscr{C}) \bigcup \mathscr{H}_{i, k}(\mathscr{C})\right)=\{z \in \mathbb{C}:|z| \leq 27.5013\} .
\end{aligned}
$$

From Theorem 2.1, we have

$$
\Upsilon(\mathscr{C})=\bigcup_{i, k \in[n], k \neq i}\left(\hat{\Upsilon}_{i, k}(\mathscr{C}) \bigcup \tilde{\Upsilon}_{i, k}(\mathscr{C})\right)=\{z \in \mathbb{C}:|z| \leq 27.5013\} .
$$

Let $\lambda^{*}$ be the largest $C$-eigenvalue of the piezoelectric tensor and $\left[-\rho_{\Gamma}, \rho_{\Gamma}\right],\left[-\rho_{\mathscr{L}}, \rho_{\mathscr{L}}\right]$, $\left[-\rho_{\mathscr{M}}, \rho_{\mathscr{M}}\right]$ and $\left[-\rho_{\Upsilon}, \rho_{\Upsilon}\right]$ are the intervals generated by Theorems $1.4,1.5,1.6$ and 2.1 , respectively. We note that in all examples, Theorem 2.2 always provides the best result among the methods tested. Table 1 lists the results obtained by methods $[3,4,15]$ and by Theorem 2.1. It indicates that $\rho_{\Upsilon}$ is more precise than $\rho, \rho_{\min }, \rho_{\Gamma}$ and $\rho_{\mathscr{M}}$. Moreover, in some cases $\rho_{\Upsilon}$ is tighter than $\rho_{\mathscr{L}}$. Thus, the $C$-eigenvalue localisation theorem obtained in this work improves the known results in $[3,4,15]$.

\section{Conclusion}

We derived a new inclusion set for localisation of the $C$-eigenvalues of piezoelectric tensors. Numerical experiments show that it is better than the known set $\Gamma(\mathscr{C})$ and is comparable or better than the sets $\mathscr{M}(\mathscr{C})$ and $\mathscr{L}(\mathscr{C})$. 
Table 1: Numerical comparison of Theorem 2.1 and the related results $[3,4,15]$.

\begin{tabular}{|c|c|c|c|c|c|c|c|c|}
\hline & $\mathscr{A}_{V F e S b}$ & $\mathscr{A}_{\mathrm{SiO} 2}$ & $\mathscr{A}_{C r 2 A g B i O 8}$ & $\mathscr{A}_{\mathrm{RbTaO3}}$ & $\mathscr{A}_{\text {NaBiS2 }}$ & $\mathscr{A}_{\text {LiBiB2O5 }}$ & $\mathscr{A}_{K B i 2 F 7}$ & $\mathscr{A}_{\mathrm{BaNiO} 3}$ \\
\hline$\lambda^{*}$ & 4.2514 & 0.1375 & 2.6258 & 12.4234 & 11.6674 & 7.7376 & 13.5021 & 27.4628 \\
\hline$\rho$ & 7.3636 & 0.2882 & 5.6606 & 30.0911 & 17.3288 & 15.2911 & 22.6896 & 38.8162 \\
\hline$\rho_{\min }$ & 7.3636 & 0.2834 & 5.6606 & 23.5377 & 16.8548 & 12.3206 & 20.2351 & 35.3787 \\
\hline$\rho_{\Gamma}$ & 7.3636 & 0.2834 & 5.6606 & 23.5377 & 16.8548 & 12.3206 & 20.2351 & 27.5396 \\
\hline$\rho_{\mathscr{L}}$ & 7.3636 & 0.2744 & 4.8058 & 23.5377 & 16.5460 & 11.0127 & 18.8973 & 27.5109 \\
\hline$\rho_{\mathscr{\mu}}$ & 7.3636 & 0.2834 & 4.7861 & 23.5377 & 16.8464 & 11.0038 & 19.8830 & 27.5013 \\
\hline$\rho_{r}$ & 7.3636 & 0.2834 & 4.7335 & 23.5377 & 16.8464 & 10.9998 & 19.8319 & 27.5013 \\
\hline
\end{tabular}

\section{Acknowledgments}

The authors are grateful to the anonymous referees for their helpful suggestions and comments.

This work was supported by the National Natural Science Foundation of China (Grants Nos. 11971413, 11571292) and by the Hunan Provincial Innovation Foundation for Postgraduate (Grant No. CX2018B381).

\section{References}

[1] C. Bu, X. Jin, H. Li and C. Deng, Brauer-type eigenvalue inclusion sets and the spectral radius of tensors, Linear Algebra Appl. 512, 234-248 (2017).

[2] C. Bu, Y. Wei, L. Sun and J. Zhou, Brualdi-type eigenvalue inclusion sets of tensors, Linear Algebra Appl. 480, 168-175 (2015).

[3] H. Che, H. Chen and Y. Wang, C-eigenvalue inclusion theorems for piezoelectric-type tensors, Appl. Math. Lett. 89, 41-49 (2019).

[4] Y. Chen, A. Jákli and L. Qi, Spectral analysis of piezoelectric tensors, J.Phys. A 51, 025206 (2017).

[5] Y. Chen, L. Qi and E.G. Virga, Octupolar tensors for liquid crystals, arXiv 1701.06761 (2017).

[6] J. Curie and P. Curie, Developpement, parpression, de lelectricite polaire dans les cristaux hemiedres a faces inclinees, Comptes rendus (in French), 91, 294-295 (1880).

[7] M. De Jong, W. Chen, H. Geerlings, M. Asta and K.A. Persson, A database to enable discovery and design of piezoelectric materials, Sci. Data 2, 150053 (2015).

[8] C. Deng, H. Li and C. Bu, Brauer-type eigenvalue inclusion sets of stochastic/irreducible tensors and positive definiteness of tensors, Linear Algebra Appl. 556, 55-69 (2018).

[9] G. Gaeta and E.G. Virga, Octupolar order in three dimensions, Eur. Phys. J. E. 39, 113 (2016).

[10] J. Jerphagnon, Invariants of the third-rank cartesian tensor: Optical nonlinear susceptibilities, Phys. Rev. B 2, 1091-1098 (1970).

[11] A.L. Kholkin, N.A. Pertsev and A.V. Goltsev, Piezolelectricity and crystal symmetry, in: Piezoelectric and acoustic materials, A. Safari and E.K. Akdog̃an (Eds), Springer (2008).

[12] I. A. Kulagin, R.A. Ganeev, R.I. Tugushev, A.I. Ryasnyansky and T. Usmanov, Components of the third-order nonlinear susceptibility tensors in KDP, DKDP and LiNbO3 nonlinear optical crystals, Quantum Electron. 34, 657-662 (2004). 
[13] C. Li, A. Jiao and Y. Li, An S-type eigenvalue localization set for tensors, Linear Algebra Appl. 493, 469-483 (2016).

[14] C. Li and Y. Li, An eigenvalue localization set for tensors with applications to determine the positive (semi-)definiteness of tensors, Linear Multilinear Algebra 64, 587-601 (2016).

[15] C. Li and Y. Li, C-eigenvalue intervals for piezoelectric-type tensors, arXiv 1704.02153 (2017).

[16] C. Li, Y. Li and X. Kong, New eigenvalue inclusion sets for tensors, Numer. Linear Algebra Appl. 21, 39-50 (2014).

[17] C. Li, J. Zhou and Y. Li, A new Brauer-type eigenvalue localization set for tensors, Linear Multilinear Algebra. 64, 727-736 (2015).

[18] L.H. Lim, Singular values and eigenvalues of tensors: a variational approach, In: CAMSAP05: Proceeding of IEEE International Workshop on Computational Advances in Multi-Sensor Adaptive Processing 1, pp. 129-132 (2005).

[19] D. Lovett, Tensor properties of crystals, IOP Publishing (1989).

[20] J. Nye, Physical properties of crystals: Their representation by tensors and matrices, Clarendon Press (1985).

[21] L. Qi, Eigenvalues of a real supersymmetric tensor, J. Symbolic Comput. 40, 1302-1324 (2005).

[22] H. Siegfried, Physical properties of crystals: An introduction, Wiley-VCH Verlag (2007).

[23] R. Varga, Geršgorin and his circles, Springer-Verlag (2004).

[24] E.G. Virga, Octupolar order in two dimensions, Eur. Phys. J. E 38, 63 (2015).

[25] G. Wang, G. Zhou and L. Caccetta, Z-eigenvalue inclusion theorems for tensors, Discrete Contin. Dyn. Syst. Ser . B 22, 187-198 (2017).

[26] W.-N. Wang, C.-X. Tang and E. Pan, Symmetric types of the piezotensor and their identification, Proc. R. Soc. A 469, 20120755-20120755 (2013).

[27] L. Zhou, J. Liu and L. Zhu, The closure property of H-tensors under the Hadamard product, Journal of Inequalities and Applications 231, 1-15 (2017). 